FORUM 2016 $31: 147$

DOI 10.1007/s12312-016-0050-8

Online publiziert: 19. Februar 2016

๑) Springer-Verlag Berlin Heidelberg 2016

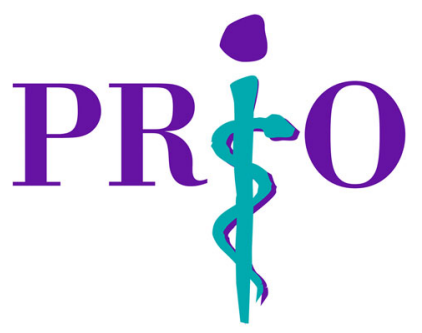

Prävention und integrative Onkologie

Jutta Hübner · AG Prävention und integrative Onkologie (PRIO)

Deutsche Krebsgesellschaft e. V., Berlin, Deutschland

\title{
Prävention von onkologischen Erkrankungen
}

\section{Fortbildung Integrative Onkologie der Arbeitsgemeinschaft PRIO}

Was beeinflusst das eigene Krebsrisiko? Was kann nach einer Krebserkrankung das Leben verlängern und die Lebensqualität verbessern? Expertinnen und Experten haben diese Themen der Prävention von Krebserkrankungen im Rahmen des vierten Seminars der Fortbildungsreihe Integrative Onkologie der Arbeitsgemeinschaft PRIO vom 29.-30.1.2016 in Wiesbaden intensiv diskutiert. Den aktuellen Stand der Wissenschaft zu Faktoren wie Rauchen, Sport, Ernährung, und Strahlung wurde hinsichtlich primärer und tertiärer Prävention erläutert. Auch die Möglichkeiten der therapeutischen Prävention wurden erklärt.

Eine Podiumsdiskussion mit Vertreterinnen und Vertretern der KrebsSelbsthilfe hat wichtige Aspekte des Survivorships beleuchtet. Die Referentinnen und Referenten besprachen ermächtigende sowie erschöpfende Erfahrungen im Leben mit und nach ihren Krebserkrankungen gemeinsam mit dem multiprofessionellen Publikum.
Nach den Vorträgen legte die erste Gruppe von Teilnehmerinnen und Teilnehmern die mündliche und schriftliche Prüfung für das Zertifikat Integrative Onkologie ab. Glückwunsch an alle zehn, die ihr Wissen und Können in den Bereichen Sport, Ernährung, Komplementärmedizin und Prävention zeigten!

\section{Nächste Termin}

Am 16. und 17.9.2016 geht die Seminarreihe der PRIO weiter. Das Thema Ernährung wird zum zweiten Mal mit Referenten aus Wissenschaft, Praxis und Selbsthilfe in Wiesbaden diskutiert.

\section{Korrespondenzadresse}

\section{PD Dr. med. J. Hübner}

Deutsche Krebsgesellschaft e.V.

Kuno-Fischer-Str. 8, 14057 Berlin, Deutschland huebner@krebsgesellschaft.de

\section{Hier steht eine Anzeige}

\section{Springer}

